\title{
CRISTARMADILLIDIUM ZARAGOZAI N. SP. (ONISCIDEA, ARMADILLIDIIDAE), UN NUEVO ISÓPODO TERRESTRE DE LA PENÍNSULA IBÉRICA
}

\author{
Julio Cifuentes ${ }^{1} \&$ Carlos E. Prieto ${ }^{2}$ \\ ${ }^{1}$ Departamento de Biología (Zoología), Facultad de Ciencias, Universidad Autónoma de Madrid, 28049 Cantoblanco, Madrid, \\ España. Email: jcifcol@gmail.com - ORCID-iD: https://orcid.org/0000-0002-9569-6973 \\ ${ }^{2}$ Departamento de Zoología y Biología Celular Animal, Facultad de Ciencia y Tecnología, \\ Universidad del País Vasco, POBox 644, 48080 Bilbao, España. \\ carlos.prieto@ehu.eus - ORCID-iD: https://orcid.org/0000-0003-2064-4842
}

\section{RESUMEN}

En este trabajo se describe una nueva especie de isópodo terrestre de la familia Armadillidiidae del levante español, C. zaragozai n. sp. Se discuten sus caracteres diagnósticos que permiten separarla de las otras dos especies del género.

\section{urn:Isid:zoobank.org:pub:3BB0B30E-BEA9-4024-981D-641D0438576D}

Palabras clave: Isópodos terrestres, Oniscidea, Armadillidiidae, taxonomía, nueva especie, península ibérica, Valencia.

\section{ABSTRACT}

Cristarmadillidium zaragozai n. sp. (Oniscidea, Armadillidiidae), a new species of terrestrial isopod from the Iberian Peninsula

A new species of terrestrial isopod belonging to Armadillidiidae family from eastern Spain, C. zaragozai n. sp., is described. Its diagnostic characteristics are discussed, and also its similarity with the other two species of the genus are discussed.

Key words: Terrestrial isopod, Oniscidea, Armadillidiidae, taxonomy, new species, Iberian Peninsula, Valencia.

Recibido/Received: 27/12/2019; Aceptado/Accepted: 23/04/2020; Publicado en línea/Published online: 01/09/2020

Cómo citar este artículo/Citation: Cifuentes, J. \& Prieto, C. E. 2020. Cristarmadillidium zaragozai n. sp. (Oniscidea, Armadillidiidae), un nuevo isópodo terrestre de la península ibérica. Graellsia, 76(2): e111. https://doi.org/10.3989/graellsia.2020.v76.266

Copyright: (C) 2020 SAM \& CSIC. This is an open-access article distributed under the terms of the Creative Commons Attribution 4.0 International (CC BY 4.0) License.

\section{Introducción}

El género Cristarmadillidium Arcangeli, 1935, de la familia Armadillidiidae Brandt, 1833, es un endemismo ibero-balear que contaba solamente con dos especies localizadas en el levante y sur de la península ibérica y en la isla de Ibiza. Ambas especies están bien caracterizadas, a las cuales se une C. zaragozai n. sp. también de la zona levantina. Nuestro objetivo es por tanto, describir la nueva especie y justificar los criterios taxonómicos que se han utilizado para su identificación y separación del resto de especies próximas.

\section{Material y métodos}

Para la elaboración del presente trabajo, además de los ejemplares "tipo" utilizados para la descripción de la nueva especie (Figs. 1-13), se han utilizado 
ejemplares de las otras dos especies para señalar sus diferencias con la que se describe. Estos ejemplares proceden de la Colección de Fauna Cavernícola del Departamento de Zoología de la Universidad del País Vasco (CFC/ZUPV); del Museo Nacional de Ciencias Naturales de Madrid (MNCN), y del Museu de Ciències Naturals de Barcelona (MZB).

Cristarmadillidium breuili Vandel, 1954 (Fig. 14-18): Alicante, Alfas del Pi, cova del Far, 21-V-2016, 2 수 y 3 우, C. Prieto leg., CFC/ZUPV 3827. Valencia, Albaida, cueva Melchor, 31-V-1976, 1 O , O. Escolà leg., MZB 70-0015.

Cristarmadillidium muricatum (Budde-Lund, 1885) (Fig. 19-21): Alicante, Agres, cueva de los Pilares, I-1936, 1 o y 1 ejemplar sin pleon, R. Verdú leg., MNCN 2004/9916. Benimantell, cova del Monedor, 23-IV-1934, 18 ふ๋̋ y 14 오, C. Bolívar leg., MNCN 2004/9896. Dénia, cueva de la Punta de Benimaquia, 23-VIII-1930,

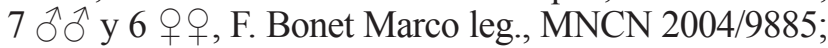
1 ㅇ, 1-VII-1984, CFC/ZUPV 67; sin fecha, 2 ô $\widehat{\jmath}$ y 1 ㅇ, E. Moroder leg., MNCN 2004/9750.

Para la elaboración de los dibujos que figuran en este trabajo, se ha utilizado una cámara digital Bresser MikroCam SP 3.1, y el editor gráfico libre InKscape (https://inkscape.org/es/).

\section{Resultados}

Género Cristarmadillidium Arcangeli, 1936

Cristarmadillidium zaragozai $\mathrm{n}$. $\mathrm{sp}$.

urn:1sid:zoobank.org:act:76FFAF5B-86E8-490E-8A1C73F11C53A766

Figs. 1-13, 22

Localidad tíPICA: Cova Xurra, Molló de la Creu, Gandia, Valencia; $38.97422^{\circ} \mathrm{N}, 0.21486^{\circ} \mathrm{W}$ [MGRS: 30 SYJ4127717605], $85 \mathrm{~m}$ s.n.m. Cavidad de $350 \mathrm{~m}$ de desarrollo y $-40 \mathrm{~m}$ de desnivel. Topografía y descripción disponibles en: http://gim-elx.epizy. com/xurra.htm.

Material típico. Holotipo: ô, 21-V-2016, C. Prieto y J. A. Zaragoza leg., MNCN 20.04/12229 [ex-CFC/ZUPV 3833] (Figs. 4-7, 10-13).

Paratipos: misma localidad y fecha que el holotipo, $6 \hat{\delta} \hat{\sigma} \mathrm{y}$ 3 웅 (Figs. 1-3, 8-9), CFC/ZUPV 3833; 2 ㅊํ y 1 우, MNCN 20.04/12230 [ex-CFC/ZUPV 3833].

Diagnosis. Coloración marrón violáceo, con el céfalon más oscuro, pero con los pleuroepímeros y las costillas blancos. Tegumento con granulaciones grandes formando costillas en el pereion en sentido antero-posterior y una fila de granulaciones en el borde posterior del pereion y del pleon. Los exopoditos de los pleópodos I y II del macho tienen forma triangular sin punta destacada de la base.
DesCrIPCIÓn. Talla holotipo: 6,5 x 2,8 mm.

Coloración: marrón violácea (Fig. 1). El céfalon es más oscuro (Fig. 2). En el pereion las inserciones musculares están muy marcadas, dejando la zona central de los terguitos más oscura (Fig. 3); los pleuroepímeros son claros (Fig. 1), el pleon es más oscuro, así como el telson. Algunos ejemplares presentan diversos grados de decoloración.

\section{Caracteres tegumentarios:}

- Granulaciones: Presenta grandes granulaciones en el vertex (Fig. 2), que en el pereion forman cinco pares de costillas en la banda media del terguito, con mayor desarrollo en los laterales, y aumentando en altura del cuarto al séptimo terguitos (Figs. 3, 8). En el borde posterior hay otra fila de granulaciones más pequeñas. En el pleon hay una fila de granulaciones muy marcada, siendo las dos centrales más grandes que el resto.

- Sedas-escamas: son pequeñas, de aspecto triangular y con las ramas basales grandes.

\section{Caracteres somáticos:}

Cuerpo muy convexo.

Ojo formado por 10 omatidios (Fig. 9).

- Céfalon de tipo elumeano (Vandel, 1954), sin la línea post-escutelar (Fig. 9), el borde posterior del escudo continua con la línea frontal, aunque hay una breve discontinuidad en su unión. El escudo cefálico es grande, ancho, y la lámina frontal cefálica sobresale ligeramente por encima del vértex.

- Pereion. El borde lateral del primer pleuroepímero está algo extendido lateralmente. No presenta schisma. El resto de pleuroepímeros caen oblicuamente.

- Pleon. Los neopleurones tercero, cuarto y quinto presentan una ligera expansión lateral y caen también oblicuamente.

- Telson triangular con el extremo redondeado (Fig. 2).

Apéndices:

Antena. El primer artejo del flagelo es casi un cuarto de la longitud del segundo. El segundo artejo de la antena lleva dos grupos de cortos estetascos.

Urópodo. El endopodito es más largo que el extremo del telson. El exopodito es casi rectangular, un poco más largo que ancho (Figs. 2, 8).

Caracteres sexuales del macho:

Pereiópodo VII sin diferenciación sexual.

Pleópodo I. Endopodito corto, fuerte (Fig. 10), y en el extremo presenta una fila de espinas en su borde interno (Fig. 4). El exopodito es triangular, con el borde posterior oblícuo por lo que la punta interna está poco individualizada de la base, y está provista de fuertes espinas (Figs. 5, 11). El campo traqueal presenta una escotadura.

- Pleópodo II. Endopodito terminado en una punta ancha (Figs. 6, 12). El exopodito es triangular, y con el campo traqueal con escotadura (Figs. 7, 13). 


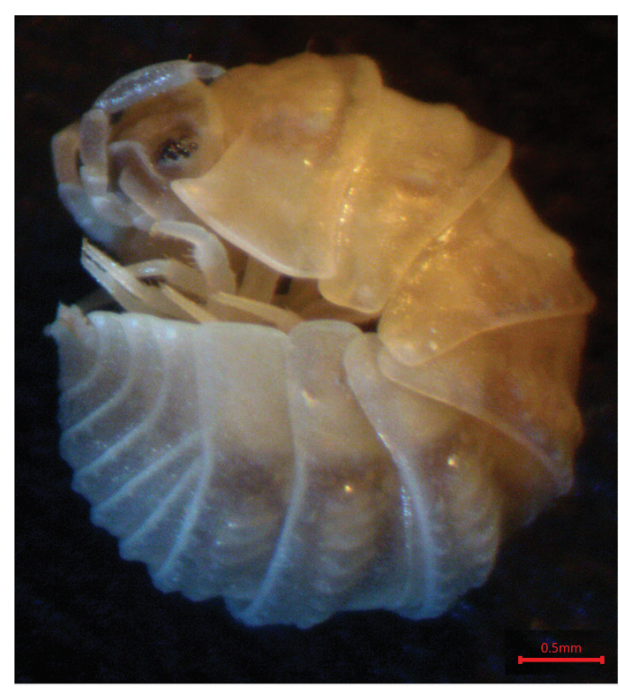

1

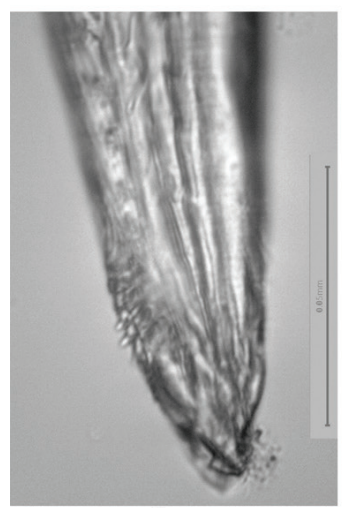

4

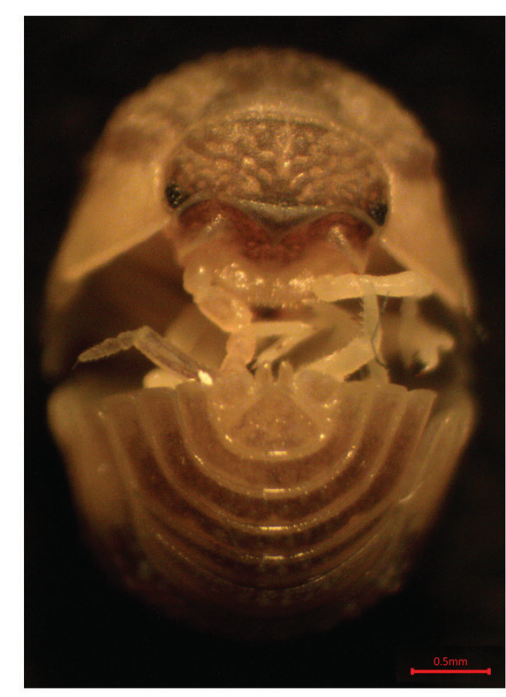

2

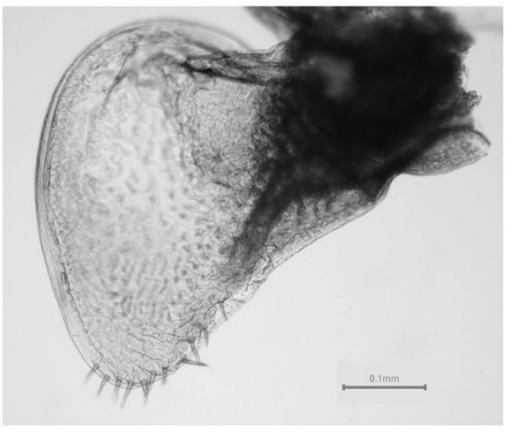

5

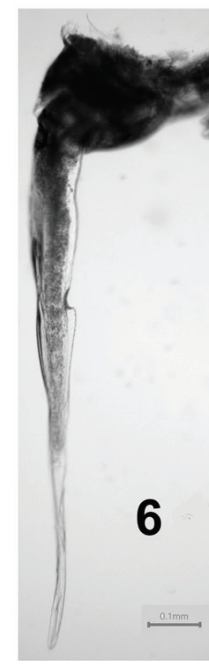

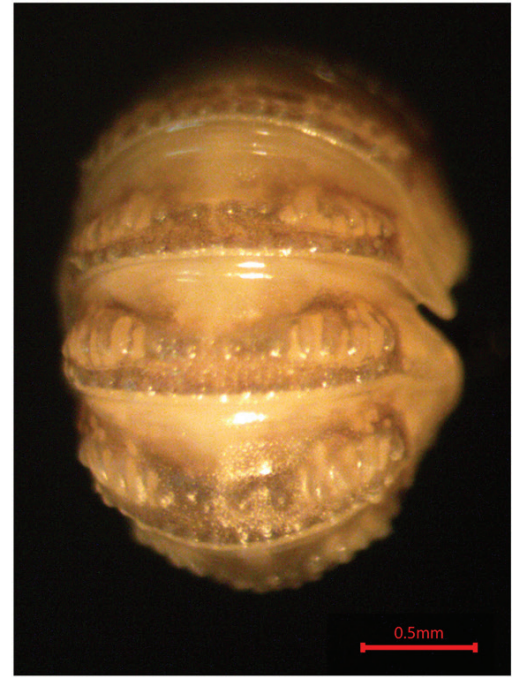

3

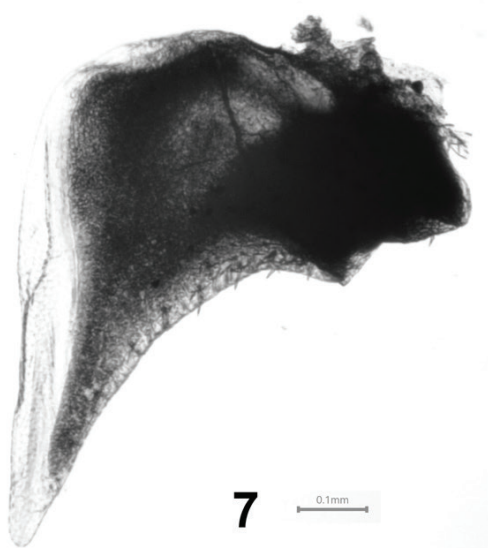

Figs. 1-7.- Cristarmadillidium zaragozai n. sp. 1: vista lateral. 2: vista del céfalon y telson. 3: vista superior. 4: endopodito I $\widehat{\delta}$

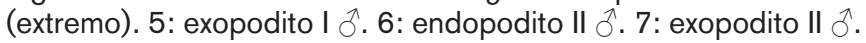

Figs. 1-7.- Cristarmadillidium zaragozai n. sp. 1: side view. 2: view of the cephalon and telson. 3: top view. 4: endopod I $\partial$

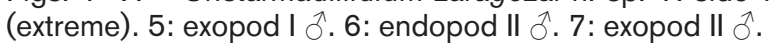

EtimologíA. Dedicada a Juan Antonio Zaragoza Miralles, activo bioespeleólogo y eminente aracnólogo (Pseudoscorpiones) en reconocimiento a su amistad y a la ayuda y compañía durante la exploración bioespeleólogica de Cova Xurra.

Ecología. Cristarmadillidium zaragozai n. sp., debe ser una especie troglófila a juzgar por las capturas realizadas, al igual que las otras dos especies del género. La biocenosis de invertebrados de la cavidad (Tabla 1) incluye también cuatro especies de gasterópodos, dos opiliones (Dicranolasma y Scotolemon n. sp., troglobio), tres colémbolos (como Troglopedetes vandeli Cassagnau \& Delamare, 1955, troglobio), un dipluro y también psocópteros, carábidos y dípteros indeterminados (observ. inéditas, C. Prieto).

\section{Discusión}

Cristarmadillidium zaragozai n. sp. es muy próxima a las otras dos especies del género, habitantes también del levante español (Fig. 22), C. muricatum, citada de Alicante (Arcangeli, 1935b; Vandel, 1954; Vives, 1982), Islas Baleares (Garcia \& Cruz, 1996), Murcia (Budde-Lund, 1885; Dolffus, 1892; Arcangeli, 1935b) y Valencia (Arcangeli, 1935b; Vandel, 1954); y C. breuili citada de Alicante (Vandel, 1954; Vives, 1982; Cruz, 1991); Córdoba y Málaga (Garcia, 2013); Jaén (Garcia, 2013; Garcia \& Pérez-Fernández, 2019), y de Valencia (González Silvestre, 2015).

Las tres especies presentan el tegumento provisto de granulaciones. En C. breuili en general son débiles 


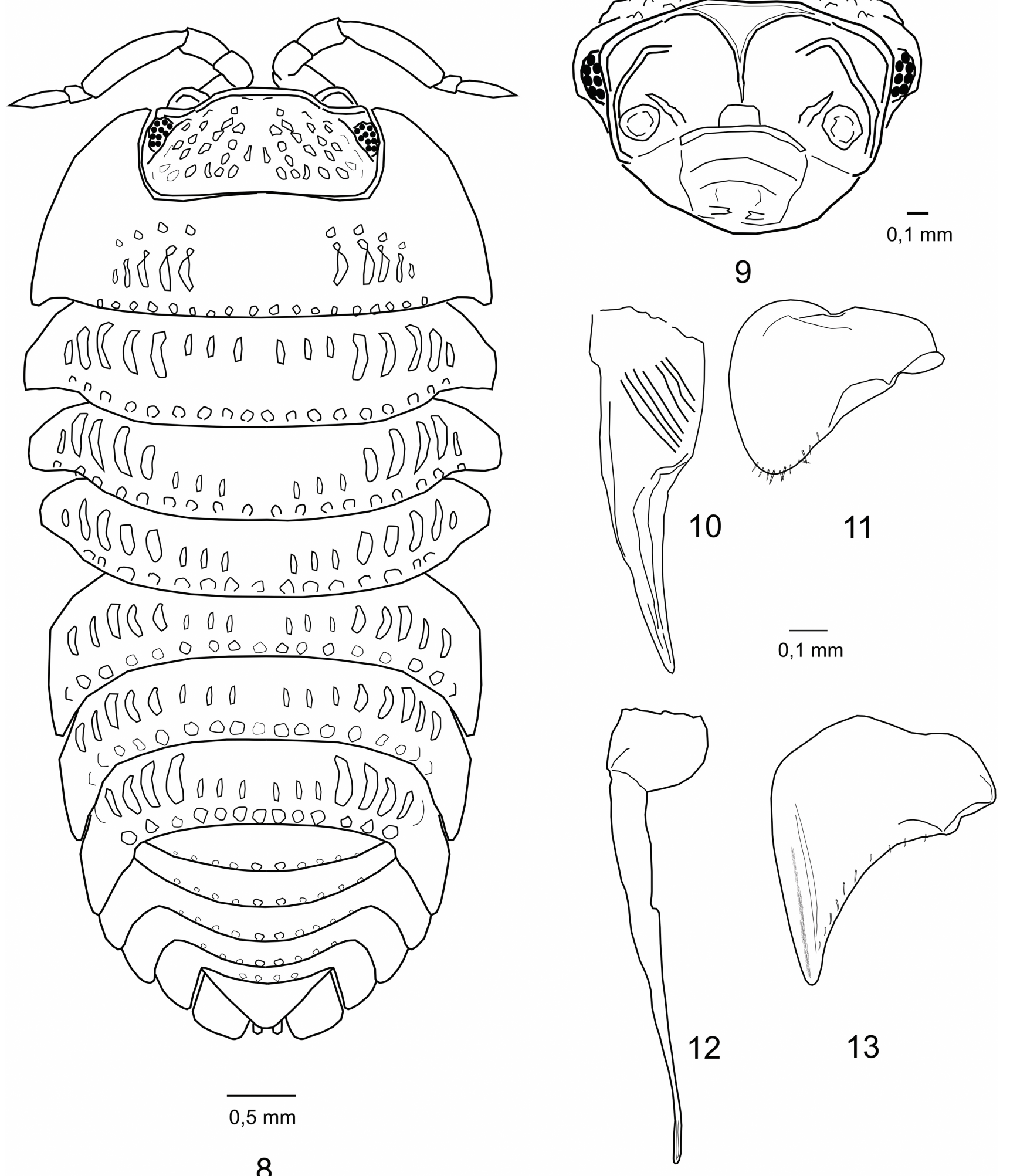

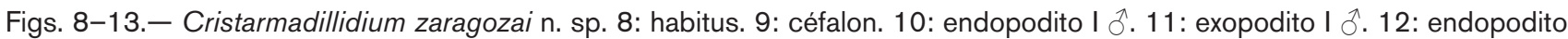
II $\hat{\jmath}$. 13: exopodito II $\hat{\jmath}$.

Figs. 8-13.- Cristarmadillidium zaragozai n. sp. 8: habitus. 9: cephalon. 10: endopod I $\widehat{\delta}$. 11: exopod I $\hat{\delta}$. 12: endopod II $\hat{\partial}$. 13: exopod II $\hat{\sigma}^{2}$. 
Tabla 1.- Taxones de la biocenosis presente en Cova Xurra.

Table 1.- Taxa of biocenosis present in Cova Xurra.

\begin{tabular}{lll}
\hline Orden & \multicolumn{1}{c}{ Especie } & \multicolumn{1}{c}{ Referencia } \\
\hline Mesogastropoda & Platyla polita polita (Hartmann, 1840) & Martínez-Ortí \& Robles (2005) \\
Stylommatophora & Oxychilus (Oxychilus) mercadali Gasull,1970 & Ríos Jiménez (2015) \\
Stylommatophora & Atenia quadrasi (Hidalgo, 1885) & Col. ZUPV [inédito] \\
Stylommatophora & Suboestophora jeresae (Ortiz de Zárate, 1962) & Col. ZUPV [inédito] \\
Opiliones & Dicranolasma soerensenii Thorell, 1876 & Col. ZUPV [inédito] \\
Opiliones & Scotolemon n. sp. & Col. ZUPV [inédito] \\
Collembola & Arrhopalites pygmaeus (Wankel, 1860) & Da Gama (2005) \\
Collembola & Mesogastrura ojcoviensis (Stach, 1919) & Da Gama (2005) \\
Collembola & Troglopedetes cf. vandeli Cassagnau \& Delamare, 1955 & Da Gama (2005) \\
Diplura & Metajapyx moroderi (Silvestri, 1929) & Sendra et al. (2006) \\
\hline
\end{tabular}

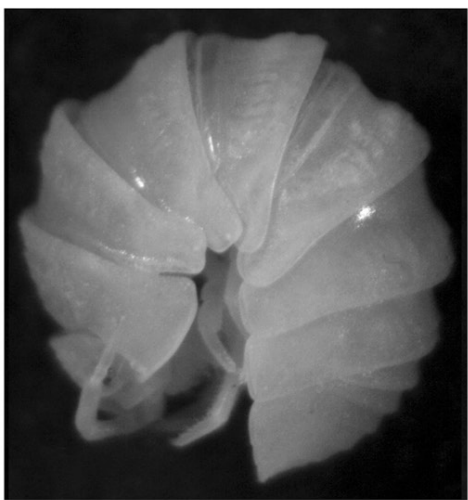

14

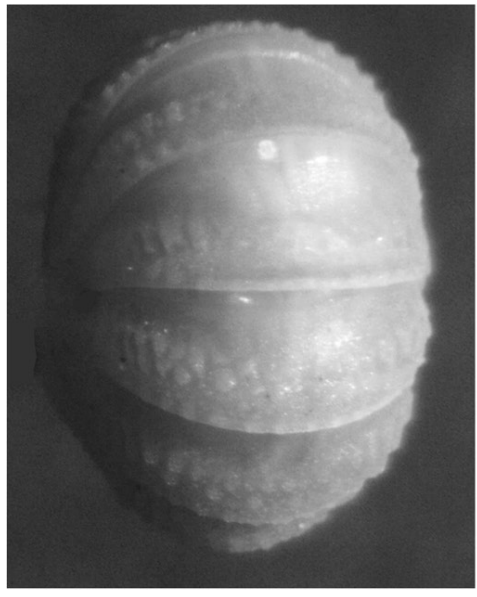

16

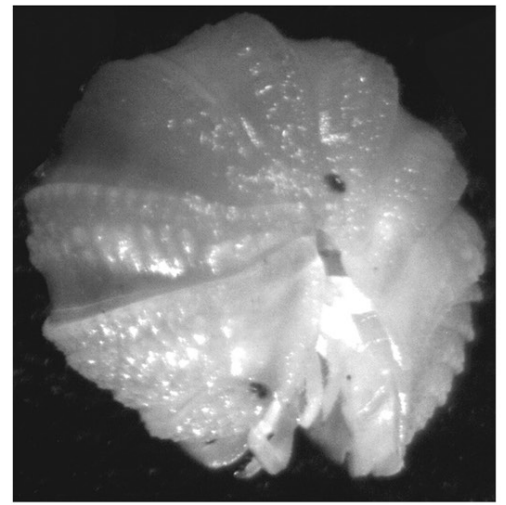

15

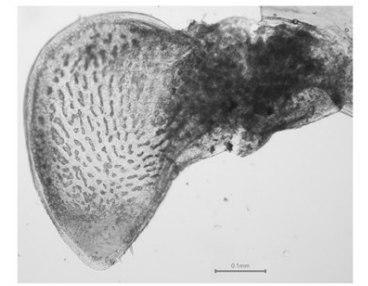

17

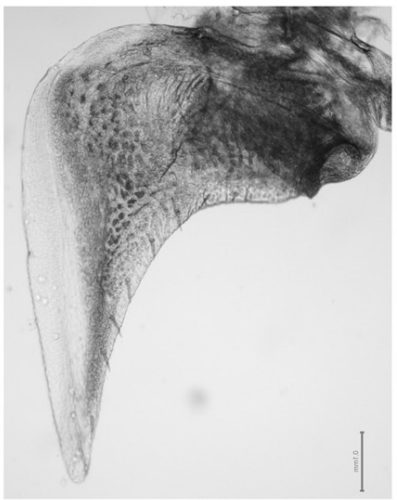

18

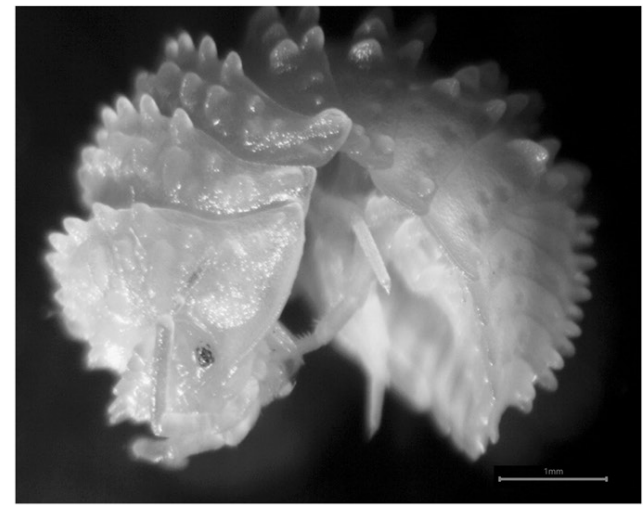

19

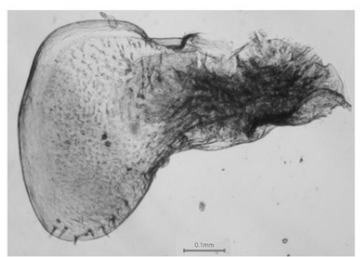

20

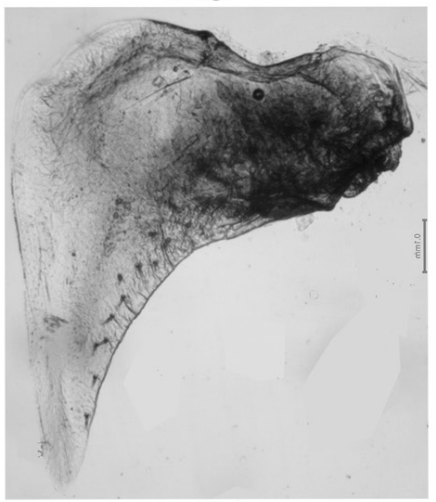

21

Figs. 14-21.- Cristarmadillidium breuili Vandel, 1954. 14: vista lateral de un ejemplar casi liso. 15: vista lateral de un ejemplar con granulaciones. 16: vista superior del ejemplar anterior. 17: exopodito I $\delta$. 18: exopodito II $\delta$. C. muricatum. 19: vista lateral. 20: exopodito I $\delta^{\lambda} .21$ : exopodito II $\delta^{\lambda}$.

Figs. 14-21.- Cristarmadillidium breuili Vandel, 1954. 14: side view of an almost smooth specimen. 15: side view of a specimen with granulations. 16: top view of the previous specimen. 17: exopod I $\partial$. 18: exopod II $\delta$. Cristarmadillidium muricatum. 19: side view. 20: exopod I $\hat{~}$. 21: exopod II ${ }^{\text {. }}$. 


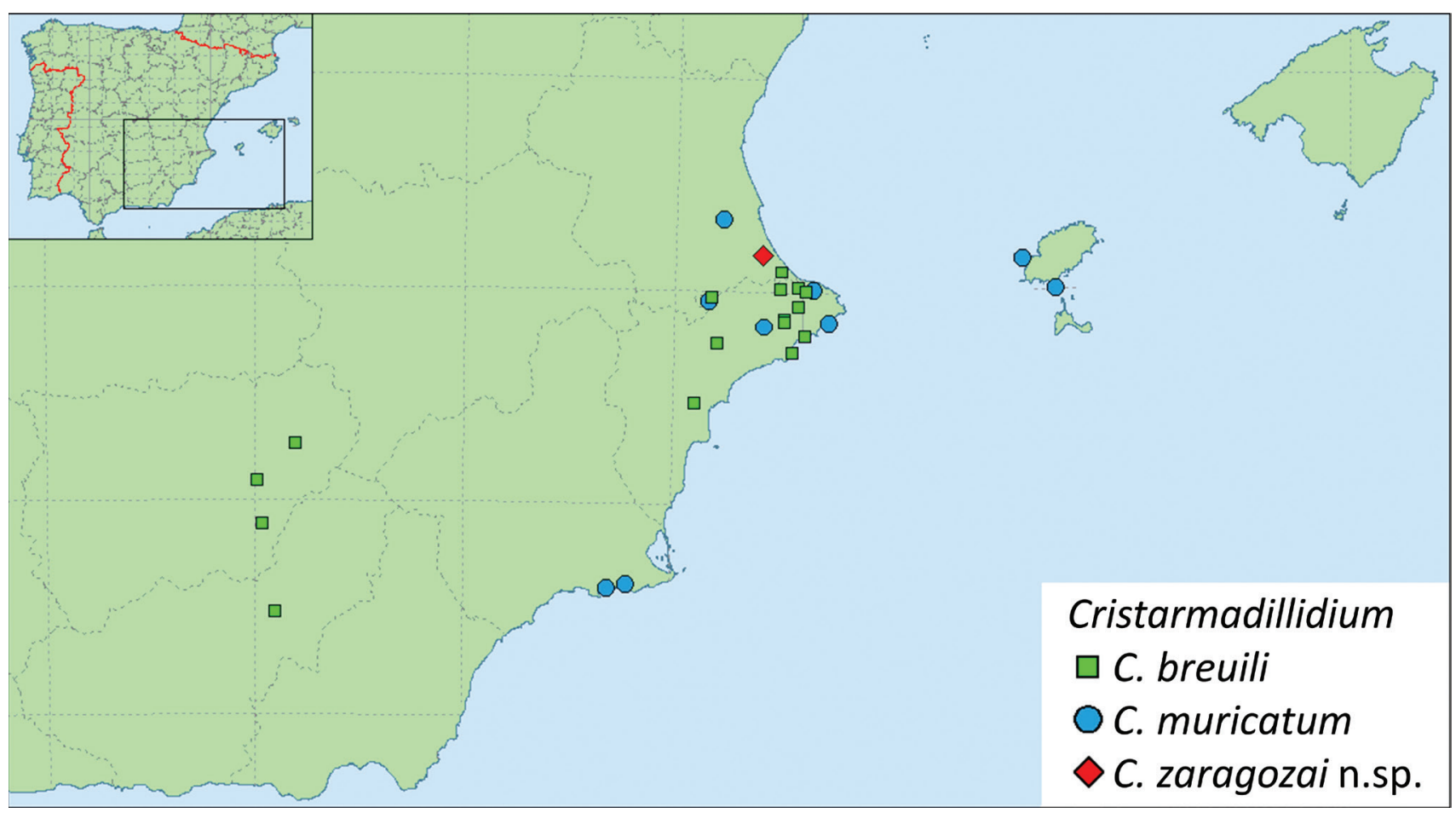

Fig. 22.- Distribución geográfica de las tres especies de Cristarmadillidium. C. breuili Vandel, 1954 sólo se ha citado de cavidades subterráneas, mientras que C. muricatum (Budde-Lund, 1885) es una especie epígea mayoritariamente litoral aunque citada de una cavidad del interior de Valencia (C. Maravillas, Alcira) y ahora encontrada en otras dos del interior de Alicante (C. Pilars, Agrés y C. Monedor, Benimantell). Garcia \& Pérez-Fernández (2019) señalan que las localidades de Andalucía podrían corresponder a una especie próxima a C. breuili.

Fig. 22.- Geographic distribution of the three species of Cristarmadillidium. C. breuili Vandel, 1954 has only been cited from underground cavities, while C. muricatum (Budde-Lund, 1885) is a predominantly coastal epigeal species although cited from a cavity of Valencia (C. Maravillas, Alcira) and now found in two others in the interior of Alicante (C. Pilars, Agrés and C. Monedor, Benimantell). Garcia \& Pérez-Fernández (2019) point out that the towns of Andalusia could correspond to a nearby species to C. breuili.

(Fig. 14), aunque en algunos ejemplares están más marcadas (Fig. 15) y en los terguitos del pereion se disponen en una banda central permaneciendo individualizadas (Fig. 16), así como una fila de débiles granulaciones en el borde posterior; en el pleon hay una fila de pequeñas granulaciones en el borde posterior. En C. zaragozai n. sp., en los terguitos del pereion las granulaciones son grandes, formando costillas en sentido antero-posterior, donde no se diferencian las granulaciones individuales (Figs. 1, 3, 8), y hay también una fila de granulaciones marcadas en el borde posterior de cada terguito; en el pleon hay una fila de granulaciones muy marcadas, con las dos centrales más grandes. En C. muricatum están hipertrofiadas (Fig. 19) tanto en céfalon como en el pereion y el pleon. Estas granulaciones permiten por tanto diferenciar fácilmente las tres especies. También presentan diferencias en la forma del exopodito I del macho, con una punta destacada de la base en C. breuili (Fig. 17) y C. muricatum (Fig. 20) aunque en esta especie es más redondeada y con fuertes espinas en el borde interno al igual que en $C$. zaragozai n. sp. (Figs. 5, 11), espinas que son muy escasas y pequeñas en $C$. breuili. Además en C. zaragozai n. sp. el exopodito I del macho tiene forma triangular, sin la punta tan marcada de las otras dos especies. En cuanto al exopodito II del macho, en C. breuili (Fig. 18) у C. muricatum (Fig. 21) presenta una punta interna muy alargada que se destaca de la base casi rectangular (Figs. 18, 21), mientras que esta punta es mucho más reducida en C. zaragozai $\mathrm{n}$. sp. (Figs. 7, 13), por lo que el exopodito II es triangular. La coloración también es diferente en las tres especies, así $C$. breuili suele estar despigmentado por lo que muchos ejemplares son blancos con los ojos negros, excepto en algunos ejemplares que presentan algunos restos de pigmentación marrón en el pereion incluidos los pleuroepímeros, y algo más oscuro en el borde posterior de los terguitos del pereion, en el pleon y en las antenas (datos propios). Cristarmadillidium muricatum también puede presentar intensa decoloración por lo que son absolutamente blancos pero con los ojos negros, pero los ejempares de vida epígea presentan una coloración parduzca (Budde-Lund, 1885; Arcangeli, 1935a). En cambio, los ejemplares de C. zaragozai n. sp. están bien pigmentados dejando los pleuroepímeros y las costillas blancos, y aunque algunos ejemplares son algo más claros, se mantiene la distribución general del pigmento. 


\section{Agradecimientos}

A Juan Antonio Zaragoza que nos ha ayudado en la captura de los ejemplares que han permitido describir la nueva especies. A la Dra. Begoña Sánchez del Museo Nacional de Ciencias Naturales de Madrid por su ayuda en el estudio de la colección de isópodos, así como a Glòria Masó, conservadora de la colección de artrópodos del Museo de Barcelona por su ayuda para la consulta de la colección de isópodos. Al Dr. Ernesto Recuero y a D. Lluc Garcia por sus valiosos comentarios que han ayudado a mejorar este trabajo.

\section{Referencias}

Arcangeli, A., 1935a. Sopra alcuni sottogeneri di Armadillidium Br. (isopodi terrestri). Bollettino dei Musei di Zoologia ed Anatomia comparata della reale Università di Torino, 45: 133-151, plates 1-4.

Arcangeli, A., 1935b. Isopodi terrestri di caverne della Spagna (Collezione del Museo di Storia naturale di Madrid). Eos, 10: 171-195.

Budde-Lund, G., 1885. Crustacea Isopoda terrestria per familias et genera et species descripta. Nielsen \& Lydiche. Copenhagen. 319 pp.

Cruz, A., 1991. Isópodos terrestres de la colección del Museu de Zoología de Barcelona (Crustacea, Oniscidea). Miscel-lánea zoológica Barcelona, 15: 81-102.

Da Gama, M. M., 2005. Collembola (Hexapoda) from caves of the Community of Valencia, Spain. Zoologia baetica, 16: 47-57.

Dollfus, A., 1892. Catalogue raisonné des isopodes terrestres de l'Espagne. Anales de la Sociedad española de Historia natural, 21: 161-190.
Garcia, L., 2013. Isópodos terrestres (Crustacea: Oniscidea) recolectados en cavidades subterráneas de Jaén. In: Pérez Fernández, T. \& Pérez Ruiz, A. (Coords.). Los invertebrados de hábitats subterráneos de Jaén. Grupo de Espeleología de Villacarrillo (G.E.V.). Jaén: 78-87.

Garcia, L. \& Cruz, A., 1996. Els isopòdos terrestres (Crustacea: Isopoda: Oniscidea) de les Illes Balears: catàleg d'espècies. Bolletí de la Societat d' Història natural de les Balears, 39: 77-99.

Garcia, L. \& Pérez-Fernández, T., 2019. Nuevos datos sobre isópodos terrestres (Crustacea: Oniscidea) recolectados en algunas cavidades subterráneas de Jaén, Granada, Málaga y Albacete. Monografias Bioespeleológicas, 14: 1-5

González Silvestre, J. V., 2015. Memorias del inframundo: Bioespeleologia I. Gota a gota, 7: 1-13.

Martínez-Ortí, A. \& Robles, F., 2005. Los caenogasterópodos terrestres (Mollusca, Orthogastropoda) de la Comunidad Valenciana (España). Iberus, 23 (2): 7-24.

Ríos Jiménez, F., 2015. Comentarios bibliográficos sobre moluscos en cavidades subterráneas de la península ibérica. Gota a gota, 9: 28-35.

Sendra, A., Ortuño, V. M., Moreno, A., Montagud, S. \& Teruel, S., 2006. Gollumjapyx smeagol gen. n., sp. n., an enigmatic hypogean japygid (Diplura: Japygidae) from the eastern Iberian Peninsula. Zootaxa, 1372: 35-52. https://doi.org/10.11646/zootaxa.1372.1.4

Vandel, A., 1954. Une nouvelle espèce cavernicole appartenant a la famille des Armadillidiidae et provenant de l'Espagne orientale (Crustacés; Isopodes terrestres). Notes biospéologiques, 9: 61-66.

Vives, M. 1982. Nota sobre la fauna d'isopodes terrestres i cavernicoles de la zona d'Alacant (Crustacea, Isopoda, Oniscoidea). Arxiu del Centre Excursionista de Terrassa (4 época), 29 (SIS/9): 8-11. 